\title{
Fabrication of high frequency SAW resonators using AIN/Diamond/Si technology
}

G.F. Iriarte (1,*), J.G. Rodríguez (1), R. Ro (2), R. Lee (2), O. A. Williams (3), D. Araujo (4), M.P. Villar (4), F. Calle(1)

(1) ISOM-ETSIT, Polytechnic University of Madrid, Spain; (2) I-Shou University, Taiwan; (3) Fraunhofer IAF, Germany, and Cardiff University, UK;

(4) University of Cadiz, Spain

(*)E-mail: gonzalo.fuentes@upm.es

\section{Background, Motivation and Objective}

High-frequency surface acoustic wave (SAW) devices are needed more and more as the demand of large volume data transmission is required. In order to increase the frequency we can either decrease the wavelength, which depends on the size of the interdigital transducers (IDTs), or increase the phase velocity, which depends on the material. The first option entails the introduction of e-beam lithography on the manufacturing process. The alternative choice requires a careful selection of a piezoelectric layer and a substrate with a high phase velocity. The AlN/diamond bi-layer system is a perfect combination for high-frequency SAW devices. Diamond is an ideal candidate due to its sound velocity, around 11.000 $\mathrm{m} / \mathrm{s}$. Furthermore, diamond shows several advantages for MEMS (micro-electro-mechanical systems) on Si in terms of Young modulus (1300 GPa for diamond and below $200 \mathrm{GPa}$ for $\mathrm{Si}$ ) or friction coefficient [1] Recent progresses in the growth of diamond by CVD (chemical vapor deposition) allowed to control the incorporation of impurities and dopants [2] and the microstructure (nanocrystalline diamond, NCD and ultra-nano-crystalline diamond UNCD [3]), both affecting the acoustic behavior of the material. This permits the development of devices oriented to biological [4], aggressive [5] or high radiative [6] environments. Thus, the AlN/diamond bilayer structure together with e-beam lithography processing is a perfect combination to increase the operating frequency of surface acoustic wave
(SAW) devices for cell phones, satellite services and wireless systems.

\section{Statement of Contribution/Methods}

AlN thin films have been deposited at room temperature using a home-built reactive sputtering system. For process details please refer to [7]. The films were synthesized on top of polished micro and nanocrystalline diamond substrates in order to process high frequency devices. Nanocrystalline diamond (NCD) films are grown by chemical vapor deposition on Si substrates. Nevertheless, the principal disadvantage these NCD films is their high rough surfaces, that degrades the piezoelectric film quality and hence the SAW response. In this work we present different approaches to achieve high frequency SAW devices on NCD/Si substrates, namely: smoothing the AlN surface by different methods or removing the $\mathrm{Si}$ substrate to use the back side diamond surface. We also propose herewith a method to wet-etch a small area of the Si substrate and thereafter deposit AlN on the backside of the free-standing diamond.

In order to get well oriented $\mathrm{AlN}$, the diamond substrates have to be planarized as the high surface roughness worsens the quality of the sputtered piezoelectric film. Both chemical-mechanical polishing and $\mathrm{SF}_{6}-\mathrm{O}_{2}$ reactive ion etching have been used to smooth the NCD. The AlN film has also been grown on the nucleation face of the diamond film once the $\mathrm{Si}$ substrate has been etched out (diamond 
freestanding). $\theta / 2 \theta$ and rocking curve X-ray diffraction analysis were used to determine the crystallographic quality of the AlN thin film. The surface roughness has been measured using an atomic force microscope (AFM). Conventional transmission electron microscopy (CTEM) analysis in electron diffraction mode was performed for structural characterization on cross-sectional prepared samples using a Jeol 1200 EX TEM.

Finally, SAW one port resonators have been fabricated on a polished sample (P) and two freestanding films with high (FH) and small (FS) surface roughness. E-beam lithography has been used to manufacture IDT on AlN/diamond. The electrical characterization of these devices (in terms of S11 parameters) is reported. The measured data has been fitted using analytic and FEM based methods.

\section{Results}

The synthesis of AlN on diamond is a great challenge, not only because of the between an AlN/diamond interface, but also because of the high surface roughness of the diamond layers [8, 9]. In the case of microcrystalline diamond, the last problem was solved by polishing. However, polishing nanocrystalline diamond is not straightforward. For the diamond synthesis by CVD, silicon was used as a substrate. The diamond/Si interface presents a smoother diamond than the diamond/air interface.
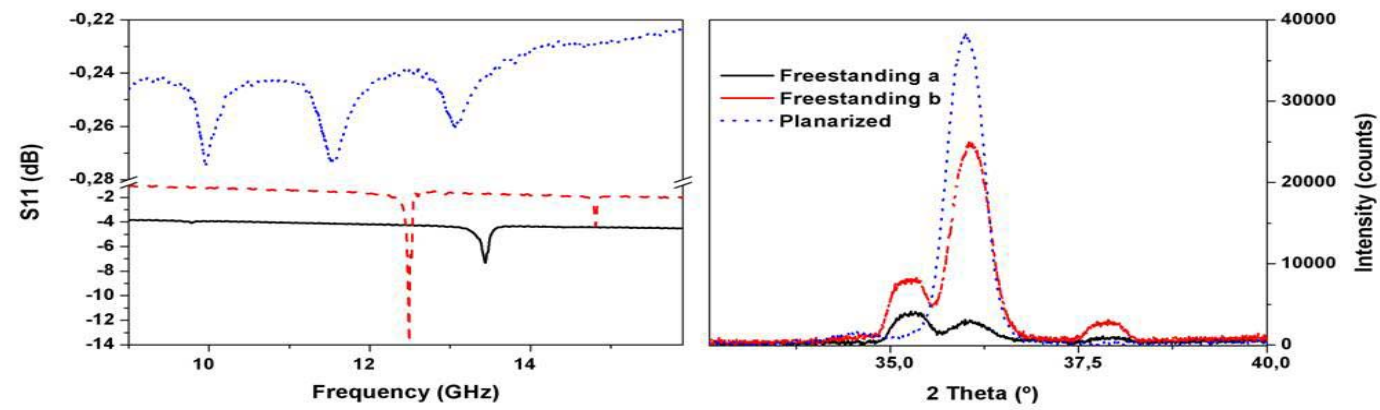

Fig.1: XRD and frequency response of SAW devices on different AlN/diamond structures

The crystallinity of the polished NCD film is even better than that of the freestanding ones, as shown in Fig. 1(right). However, the device provides a worse SAW response, as shown in Fig. 1(left) where the $S_{11}$ responses of the three different SAW resonators are compared.SAED (selective area electron diffraction) patterns are shown in figure 2 . Three of them are recorded at different height in the AlN layer and a dashed circle show the location of the probed region on the micrograph when introducing the selective area aperture (corresponding, after the electron optics, to a diameter of $500 \mathrm{~nm}$ on the sample). Significative differences are revealed depending on the location of the aperture: (i) the first SAED at the diamond-AlN interface show arc-shaped spots (part of circle) corresponding to $20^{\circ}$ grain misorientation;(ii) at the middle of the $1.85 \mu \mathrm{m}$ thick layer (at around $800 \mathrm{~nm}$ from the diamond-AlN interface), those arcs are reduced down to $6^{\circ}$ grain misorientation. Only some spots are observed to correspond to each reflection, indicating that just 4-6 grains are irradiated by the electron beam; (iii) near the free surface at the top of the AlN layer the spots are surprisingly punctual and well defined, that seems to indicate a monocrystalline structure. Indeed, the DF micrograph shows grains with size down to 
10x100 nm while others can reach 100x300 nm. Thus with considered aperture both observations are consistent in determining the grain size at the interface. At the center of the AlN layer, only 4-6 grains participate to the spot formation; that means larger grains with more accurate orientation as the arc size is reduced. Grains are larger and well oriented at the top of the layer near the surface. As a result, micrographs of fig.2 (left) and fig. 2 (middle) indicate that only 2-3 grains can be irradiated in this region near the surface by the electron beam below the aperture, even though, only one spot is observed for each reflection (see

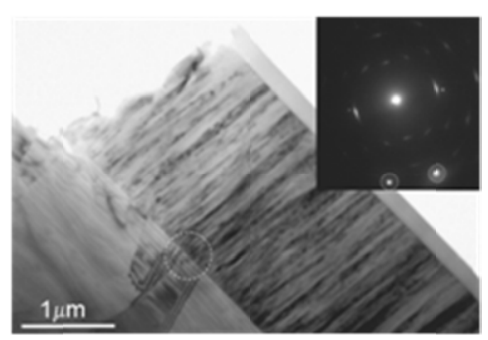

SAED of fig.2c in contrast to those of fig.2a and b). This means that those 2-3 grains are perfectly oriented. In addition, their size is also larger than at the center of the layer. Finally, fig.2 (right) shows the grain lattice orientation with respect to that of the AlN-diamond interface orientation. Indeed, this is a very important aspect to investigate, since c-axis (and thus polarity) of the piezoelectric layer, should be perpendicular to the interface to allow a piezoelectric effect capable to generate acoustic waves in the diamond crystal.

Fig.2: Selective area electron diffraction pattern (SAED) at different heigth of the AlN layer. The dashed circles indicate the position of the aperture.

A critical aspect of the AlN layers deposited by reactive sputtering is their surface roughness, as it may lead to an increase of propagation losses. This roughness is related to the surface roughness of the substrate, in this case CVD (chemical vapor deposition) synthesized diamond. We have studied the influence of the diamond substrate roughness on the AlN growth. We deposited AIN on NCD from 3 different wafers (average roughness of $10.71 \mathrm{~nm}, 6.70 \mathrm{~nm}$ and $5.85 \mathrm{~nm} \mathrm{rms}$ ) and a polished MCD wafer (average roughness of 3.70 $\mathrm{nm}$ rms).

Figure 3 shows the $S_{11}$ parameter vs. frequency for samples \#1 (left) and \#2 (right). The input impedance of SAW devices depends on lots of factors such as overlap width, number of electrodes, coupling coefficient, reflectivity, etc. The sample \#2 shows a sharp dip around mode 1, which illustrates that the input impedance of the sample around mode 1 approaches the source impedance, 50 ohm. Sample \#2 has a slightly higher velocity than the other sample, but with a small coupling coefficient, which cannot be used to design wide-band filter.

For SAW applications, we wish the resistance $\mathrm{R}$ to be zero. Both samples have comparable phase velocity. Based upon this point, the performance of sample \#1 is much better than sample \#2. The corresponding fitted polynomials and the rest COM parameters of mode 1 for both samples have been calculated (to be published). Respective plots of $S_{11}$ parameter, conductance (G) and susceptance (B) vs. frequency for both measured and fitted date will be presented. We have also extracted the resistance (R) and capacitance (C) values for samples \#1 and \#2 with different AlN thickness. We are focusing on adjusting COM parameters to fit measured G, B, 
and $\mathrm{S}_{11}$ around mode 1

The results are calculated numerically using Fortran software. The FEM data are calculated using 4 resonance frequencies as shown in Hashimoto's book [10]. The extracted data are fitted parameters from the measured data. The values of $\mathrm{C}$ and $\mathrm{R}$ are calculated first. The values

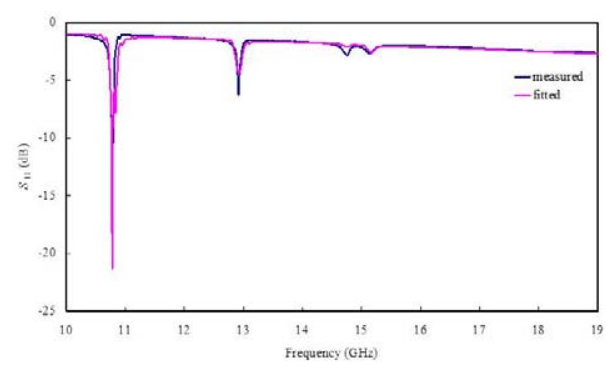

of $\mathrm{V}_{\mathrm{eff}}, \mathrm{K}^{2}, \mathrm{k}_{12}$, and loss are then fitted altogether to get best matched results. The corresponding equations will be presented.

Fig. 3: $S_{11} v s$. frequency for the sample \#1 (left) and resistance of electrode (R) of \#1 \& \#2 (right).

\section{Discussion and Conclusions}

The AlN has been synthesized on top of the diamond substrate by reactive sputtering at room temperature. The diamond layer was deposited by the chemical vapor deposition (CVD) method. SAW devices with resonant frequencies above 10 $\mathrm{GHz}$ and with more than $20 \mathrm{~dB}$ insertion loss have been manufactured using AlN films deposited by sputtering on polished and freestanding NCD films. These devices exhibit an outstanding performance in terms of IL (insertion loss) and other frequency response parameters. TEM related analysis showed that (i) the sputtering step allowed to control the AIN crystallographic orientation to have the c-axis perpendicular to the diamond-AlN interface, (ii) the microcrystalline diamond growth has $\mathrm{sp}^{2}$ bonds below the EELS detection limit with mm-range grains and, finally, (iii) that the polishing step ensure a totally flat AlN_diamond interface down to the nm-scale.

However, it is not the crystal quality, but mainly the surface roughness that is a critical structural factor determining device performance. An improved device performance in terms of insertion losses, contrast and quality factor is

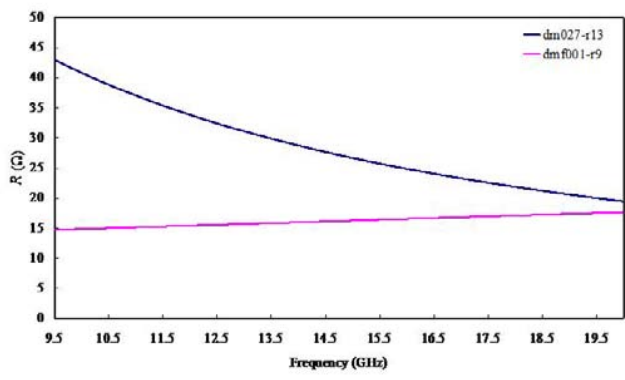

achieved for the freestanding film of higher crystallinity.

\section{References}

[1] O. Auciello S. Pacheco, A.V. Sumant, C. Gudeman, et. al., IEEE Microwave Mag. 8 (2007) 61.

[2] D. Araújo, P. Achatz, R. El Bouayadi, A.J. García, et. al., Diam. Rel. Mat. 19 (2010) 972.

[3] O. Williams, M. Nesladek, M. Daenen, et. al., Diam. Rel. Mat. 17 (2008) 1080.

[4] C. E. Nebel, B. Rezek, D. Shin, H. Uetsuka and N. Yang, J. Phys. D: Appl. Phys. 40 (2007) 6443.

[5] A. Brambilla et al. NIM-A 458, (2001) 220-6 ; P. Bergonzo et al. Diam. Rel Mat, 9 (2000) 1003.

[6] J. Morse, M. Salome, E. Berdermann, M. Pomoroski, et. al., Diam. Rel. Mat. 16 (2007) 1049.

[7]| G.F. Iriarte, J.G. Rodríguez, and F. Calle, Materials Research Bulletin, vol. 45 pag. 1039- 1045, 2010.

[8] Cheng-Hsien Chou, Yung-Chen Lin, et.al. Diamond and Related Materials 15, pp. 404-409, 2006.

[9] O. Elmazria, F. Bénédic, M. El Hakiki, et. al. Diamond and Related Materials 15, pp. 193-198, 2006.

[10] Surface Acoustic Wave Devices in Telecommunications: Modeling and Simulation, Ken-Ya Hashimoto, Springer, 2000

\section{Acknowledgments}

The authors would like to acknowledge the support of this work by Ministerio de Ciencia e Innovación, under ReADi (TEC2010-19511) project and the Acción Integrada DE2009-0015. 